\title{
Gestão de Dados de Pesquisa: um desafio para os Programas de Pós-Graduação em Enfermagem
}

\author{
Research Data Management: a challenge for Graduate Nursing Programs
}

\author{
Tatiana Souza da Silva Werle ${ }^{1^{*} \bullet}$, Iana Paes d'Assumpção Vital ${ }^{1}$, Rodrigo Viegas Moure ${ }^{1} \bullet$, Vânia \\ Andréa Schenkel Meireles ${ }^{1}$, Debora Daniela Eira Guidi ${ }^{1}$, Danielle Guaudino de Paula ${ }^{2}{ }^{\bullet}$, Patricia \\ Henning $^{2} \theta$, Viviane Veiga $^{30}$

\begin{abstract}
${ }^{1}$ Mestrandos do Programa de Pós-Graduação em Saúde e Tecnologia no Espaço Hospitalar da Universidade Federal do Estado do Rio de Janeiro, Rio de Janeiro, Brasil. ${ }^{2}$ Professor titular do Programa de Pós-Graduação em Saúde e Tecnologia no Espaço Hospitalar da Universidade Federal do Estado do Rio de Janeiro, Rio de Janeiro, Brasil. ${ }^{3}$ Professor convidado do Programa de Pós-Graduação em Saúde e Tecnologia no Espaço Hospitalar da Universidade Federal do Estado do Rio de Janeiro, Rio de Janeiro, Brasil.

*Autor para correspondência. E-mail: tatissil@uol.com.br
\end{abstract}

Resumo: Introdução: A ciência aberta abre novos horizontes tanto para o pesquisador quanto para as atividades acadêmicas e científicas. Ela vem crescendo em todas as áreas do conhecimento e chega ao domínio da enfermagem como um grande desafio a ser enfrentado. Este artigo tem o objetivo de mostrar como os Programas de Pós-Graduação em Enfermagem, da Universidade Federal do Estado do Rio de Janeiro estão lidando com essa questão e a direção que vêm tomando para realizar a devida gestão dos dados gerados em suas pesquisas, no âmbito da ciência aberta. Revisão e Discussão: Para tal fim foi necessário; contextualizar o conceito de ciência aberta, enfatizando as práticas de gestão de dados; apresentar o ciclo de vida dos dados, os princípios FAIR e sua aplicação na rede GO FAIR Brasil Saúde - Enfermagem criada com a finalidade de elaborar estratégias para a implementação dos princípios FAIR no campo da enfermagem, visando o, compartilhamento e reuso desses dados em novas pesquisas. Considerações finais: A coordenação dos Programas de Pós-graduação em Enfermagem da UNIRIO e da Escola de Enfermagem Alfredo Pinto, da Rede GO FAIR Brasil Saúde - Enfermagem representa um exemplo de adoção de práticas da ciência aberta dentro de cursos de pós-graduação. Portanto, espera-se que por intermédio dos estudos e eventos que se iniciam no âmbito da Rede GO FAIR Brasil Saúde - Enfermagem, realizados em 2020 e programados para os anos seguintes, responder à pergunta feita na introdução deste artigo e contribuir para a promoção e implementação de estratégias de capacitação de aluno e profissionais da área da saúde, em geral, sobre a gestão de dados de pesquisa na área da enfermagem.

Palavras-chave: enfermagem, ciência aberta, dados abertos, princípios FAIR, GO FAIR.

\begin{abstract}
Introduction: Open Science opens new horizons for the researcher and for academic and scientific activities. It has been growing in all areas of knowledge and comes in the nursing domain as a huge challenge to be faced. This article aims to show the path that the Nursing Postgraduate Programs at the Federal University of the Rio de Janeiro State are following to achieve a proper data management of their research, align to the scope of open science. Review and Discussion: For this purpose, it was necessary; contextualize the concept of open science emphasizing data management practices; presents the data life cycle, the FAIR principles and their application in the GO FAIR Brazil Health - Nursing network created with the purpose of elaborating strategies for the implementation of the FAIR principles in the nursing field, aiming the sharing and reuse of these data in new research. Final considerations: The coordination of the Graduate Nursing Programs at UNIRIO and the Alfredo Pinto Nursing School, from the GO FAIR Brazil Saúde Network - Nursing represents an example of the adoption of open science practices within graduate courses. Therefore, it is expected that through the studies and events that begin within the scope of the GO FAIR Brazil Health - Nursing Network, carried out in 2020 and scheduled for the following years, answer the question asked in the introduction of this article and contribute to the promotion and implementation of training strategies for students and health professionals, in general, on the management of research data in the field of nursing.
\end{abstract}

Keywords: nursing, open science, open data, FAIR principles, GO FAIR. 


\section{Introdução}

A ciência aberta, que tem como principal objetivo dar acesso ao conhecimento científico sem qualquer barreira social, econômica ou legal, para que todas as pessoas possam acessá-lo, distribuí-lo e reusá-lo em novas pesquisas, não é mais novidade dentro das comunidades científicas de todo o mundo.

Dentre as suas principais práticas, os dados de pesquisa vêm se firmando, em todo o mundo, desde que a Comissão Europeia passou a exigir, em julho de 2016, a publicação do resultado das pesquisas, por ela financiada, em acesso aberto e, um plano de gestão de dados em seu principal programa de financiamento na época, denominado Horizonte 2020 (European Commission, 2017).

Com o desenvolvimento das tecnologias de informação, aliado ao movimento da ciência aberta, a produção de dados de pesquisa tomou novas dimensões colocando-se em um nível de importância tal, que demanda uma melhor gestão e preservação, buscando não apenas a organização desses dados, mas também a possibilidade de compartilhá-los e reusá-los em novas pesquisas.

Por essa razão, a gestão apropriada dos dados de pesquisa se faz cada vez mais presente considerando a complexidade e a especificidade das áreas e disciplinas cobrindo todo o seu "ciclo de vida", desde a sua coleta até o armazenamento de longo prazo, passando por uma série de processamentos de limpeza, anotação, indexação, curadoria e transformação (Carvalho \& Barbosa Neto, 2020).

Vários institutos de pesquisa e universidades brasileiras já incorporaram a ciência aberta nas suas atividades de pesquisa e acadêmicas, por intermédio de investimento no acesso à informação científica, por meio dos periódicos de acesso abertos e dos repositórios de informação abertos. No entanto, no que diz respeito à abertura dos dados de pesquisa são poucos aqueles que assumiram tal desafio e responsabilidade (IBICT, 2018).

Nos Estados Unidos, a gestão de dados de pesquisa dentro dos cursos de pós-graduação em enfermagem já é uma realidade. Um levantamento recente sobre as práticas na educação em gestão de dados, em programas de doutorado em enfermagem, realizados naquele país, mostra um panorama promissor para a área e aponta a necessidade de adoção de uma cultura de dados que aborde todos os estágios do ciclo de vida dos dados, assim como o investimento nas habilidades do corpo docente, sobre essa temática, para poder melhor orientar os alunos na criação de pesquisas focadas em dados (Raszewski et al., 2021).

A Universidade Federal do Estado do Rio de Janeiro (UNIRIO) já deu seus primeiros passos nessa direção ao lançar, em outubro de 2018, a sua "Política de Acesso Aberto à Informação Técnico - Científica e aos Dados de Pesquisa”. Resolução nº 5.055, de 10 de outubro de 2018 (Brasil, 2018).

Por outro lado, os Programas de Pós-graduação em Enfermagem da UNIRIO vêm assumindo o protagonismo ao trazer para a área de enfermagem brasileira iniciativas pioneiras voltadas para estudos e pesquisas sobre gestão de dados em enfermagem, na perspectiva da ciência aberta. Além disso, vêm promovendo diversas ações de capacitação por meio de seminários, workshops e disciplinas sobre essa temática (https://www.youtube.com/playlist?list=PL8gW4sGfXQGPuEpCEAGZ4c3CSavNdl0UI).

Esse processo de valorização do acesso aos dados de pesquisa demanda um longo caminho a ser percorrido, principalmente dentro da área de enfermagem. E é dentro desse contexto que surge um questionamento que merece ser considerado. Como os cursos de pós-graduação em enfermagem da UNIRIO estão se preparando para a gestão dos dados de pesquisa que produzem? Para responder tal indagação foi necessário, antes de tudo, contextualizar o conceito de ciência aberta dando maior ênfase à prática da gestão de dados; apresentar os princípios FAIR, internacionalmente considerados norteadores das boas práticas de gestão de dados e, encerrar com o relato dispondo sobre a iniciativa GO FAIR Brasil Saúde - Enfermagem, que chega ao Brasil coordenado pelos Programas de Pós-graduação em Enfermagem da UNIRIO, com o objetivo de apoiar as práticas de gestão de dados no domínio da enfermagem.

\section{Revisão e Discussão}

\section{O que é ciência aberta?}

A ciência aberta é um conceito guarda-chuva que abarca diversas práticas abertas da ciência, desde o acesso aberto à informação científica, passando pela educação aberta e recursos educacionais abertos, pelos dados científicos abertos, hardware e software abertos, ciência cidadã e pelos cadernos de pesquisa abertos.

Tais práticas representam a ideia de que o conhecimento científico deve ser compartilhado abertamente, tão cedo quanto possível, desde o início do seu processo de descoberta (Nielsen, 2012). 
A primeira manifestação organizada pela comunidade científica com a participação de representantes de diversas áreas do conhecimento, a favor do acesso aberto ocorreu na década de 1990, período em que aconteceu a crise dos periódicos científicos, por conta do aumento abusivo do valor das assinaturas, o que inviabilizava sua manutenção pelas instituições e, mais ainda, pelos pesquisadores (Cavalcanti \& Sales, 2017). Foi por meio de um evento realizado em 2002, na Hungria, com a participação da comunidade internacional de editores científicos, pesquisadores, bibliotecários e professores que resultou a "Declaração de Budapest", definindo que o acesso aberto à literatura científica revisada por pares deveria ser disponibilizado livremente na Internet, permitindo a qualquer usuário ler, fazer download, copiar, distribuir, imprimir, pesquisar ou referenciar o texto integral desses artigos (Costa, 2006).

No Brasil, o Instituto Brasileiro de Informação em Ciência e Tecnologia (IBICT) vem ao longo dos últimos anos promovendo ações para o avanço da ciência nesta direção. Em 2016, lançou o Manifesto de Acesso Aberto a Dados da Pesquisa Brasileira para Ciência Cidadã (IBICT, 2016). Este documento demonstra o valor estratégico e informacional dos dados de pesquisa, estabelecendo responsabilidades para o desenvolvimento de ações de apoio, além do incentivo à adesão e às iniciativas de Ciência Aberta. Em 2017, criou o Plano de Dados Abertos 2020-2022 (IBICT, 2020), no qual estabelece como objetivo a promoção, a ampliação e o aprimoramento da abertura de dados da instituição, garantindo a sua publicidade, transparência e eficiência na administração pública, além de facilitar o intercâmbio de dados com outros órgãos para a melhoria da gestão pública.

A ciência aberta revela uma preocupação não apenas com a publicação, abertura e transparência do resultado das pesquisas, mas principalmente com a abertura e gestão apropriadas dos dados brutos de pesquisas.

Trata-se de uma revisão ampla da literatura realizada por 2 pesquisadores independentes com proposta de abordar com profundidade o tema e responder ao objetivo proposto.

\section{Os dados de pesquisa abertos}

Os dados abertos de pesquisa é atualmente pauta prioritária nas agendas científicas nacionais e internacionais, por ampliar tanto a visibilidade e a transparência das investigações, como a capacidade de reprodutibilidade e reuso dos dados em novas pesquisas (Veiga et al., 2019).

A importância dessa abertura se dá, principalmente, pela possibilidade de compreender melhor o processo de geração do conhecimento. Desta forma, a pesquisa amplia as chances de validação e de reuso dos dados sob diferentes abordagens, tornando o processo mais democrático.

Diversos países estão definindo as suas diretrizes para que os dados de pesquisa estejam publicamente acessíveis - buscando a otimização de recursos e coleta de dados, a possibilidade de comprovar os resultados e reproduzir a pesquisa, gerar novas abordagens, discussões e conclusões acerca de um conjunto de dados, ampliar as redes de colaboração nacional e internacional e reduzir o tempo para que os avanços da ciência sejam possíveis (Torino et al., 2020).

É possível afirmar que os dados de pesquisa têm uma vida maior do que os próprios projetos que os originam, tendo um ciclo de vida bem característico. Existem vários modelos de ciclo de vida dos dados de pesquisa. O mais conhecido é o modelo criado pela cooperação internacional DataONE, apoiada pela US National Science Foundation (Bertin et al., 2017), apresentado no (Quadro 1).

Quadro 1. Modelo DataONE.

\begin{tabular}{|c|c|}
\hline Etapas & Ação \\
\hline $\begin{array}{c}\text { Planejament } \\
\text { o }\end{array}$ & Objetivos do projeto, impacto, resultados e produtos esperados, um plano de gestão de dados e a política de backup \\
\hline Coleta & Estratégia para a coleta e armazenamento, descrição de como os dados são estruturados \\
\hline Validação & Descrição dos processos que garantem a qualidade dos dados \\
\hline Descrição & De quem, quando, o quê, porque, onde e como os dados foram adquiridos, permitindo o reuso e compartilhamento \\
\hline Preservação & Curto e longo prazo, permitindo o resgate das informações \\
\hline Descoberta & Localização, obtenção ou recuperação dos dados \\
\hline Integração & Combinação de dados de diferentes fontes \\
\hline Análise & Os dados são analisados por diferentes sujeitos, dando origem a resultados que levarão a um novo ciclo de \\
planejamento
\end{tabular}

Fonte: Bertin et al. (2017). 
A Comissão Europeia teve um papel fundamental neste processo de criação e gestão de dados. Desde 2017, passou a exigir um plano de gestão de dados alinhado aos princípios FAIR, em todos os estudos financiados por ela. Dessa forma, propõe uma mudança de paradigma para a área da pesquisa científica europeia, modificando o modo de pensar, operar e agir, tornando a pesquisa mais colaborativa, possível de ser compartilhada, visando à reutilização dos dados de pesquisa (Henning, Ribeiro, Sales, et al., 2019).

No Brasil, o tema ainda é recente e poucas são as exigências para a publicação em acesso aberto e a gestão dos dados de pesquisa. A Fundação de Amparo à Pesquisa do Estado de São Paulo (FAPESP) foi pioneira quando passou a exigir, em 2017, a apresentação de um plano de gestão de dados em algumas modalidades de chamadas de seus programas de fomento (Monteiro \& Lucas, 2019). A Fundação Oswaldo Cruz é a instituição de pesquisa brasileira que vem exercendo um papel de inovação em várias ações de ciência aberta no Brasil. Em 2011 institucionalizou o seu Repositório Institucional denominado ARCA (FIOCRUZ,2021) onde armazena toda a produção intelectual produzida pelos pesquisadores, professores e servidores da instituição e vem trabalhando há mais de um ano no desenvolvimento de uma ferramenta para a geração de Planos de Gestão de dados, alinhada aos princípios FAIR, para seus alunos e pesquisadores (Veiga et al., 2019).

Nesse contexto, os princípios FAIR são fundamentais por estabelecerem orientações basilares e norteadoras na gestão, curadoria e preservação dos dados de pesquisa direcionados para o compartilhamento e o reuso.

\section{Princípios FAIR e sua importância na gestão de dados}

Os princípios FAIR (Quadro 2) foram criados em 2014, durante a conferência internacional "Jointly designing a Data FAIRPORT“, na Holanda, onde estudiosos de várias áreas do conhecimento criaram critérios para análise dos dados de pesquisa com relação à sua utilização, tratamento e reuso no meio digital (Henning, Ribeiro, Sales, et al., 2019; Wilkinson et al., 2017).

Quadro 2. Definição dos princípios FAIR.

\begin{tabular}{|c|c|c|}
\hline Iniciais & Princípio & Diretrizes \\
\hline F & Findable & $\begin{array}{c}\text { Os dados devem ter identificadores globais, persistentes e que o identifiquem, ser enriquecidos de } \\
\text { identificadores que permitam ser encontrados; incluir claramente e explicitamente os caracteres } \\
\text { que o identifiquem; ser registrados ou indexados em recursos que ofereçam possibilidade de busca }\end{array}$ \\
\hline A & Accessible & $\begin{array}{c}\text { Os dados devem ser recuperáveis pelos seus identificadores usando protocolo de comunicação } \\
\text { padronizado }\end{array}$ \\
\hline I & Interoperable & $\begin{array}{c}\text { A interoperabilidade garante que os dados devem ser representados por meio de uma linguagem } \\
\text { formal, acessível, compartilhada e amplamente aplicável para a representação do conhecimento } \\
\text { usando vocabulários que seguem os princípios FAIR e finalmente os (Meta) dados devem incluir } \\
\text { referências qualificadas para outros (Meta) dados }\end{array}$ \\
\hline R & Reusable & $\begin{array}{c}\text { Nesta etapa serão definidas as especificações dos (Meta) dados descritos com uma pluralidade de } \\
\text { atributos precisos e relevantes com disponibilização de licenças de uso claras e acessiveis os dados } \\
\text { devem estar associados à sua proveniência e devem estar alinhados com padrões relevantes ao seu } \\
\text { domínio }\end{array}$ \\
\hline
\end{tabular}

Fonte: Wilkinson et al. (2017).

Os princípios FAIR, para serem implementados, devem ser bem planejados observando um ambiente sustentável, garantindo que os dados e serviços de apoio sejam realmente FAIR; além de desenvolver políticas que promovam e estimulem a criação destes princípios (Henning, Ribeiro, Sales, et al., 2019). Para que isso aconteça de forma organizada e sustentável, várias iniciativas sugiram no mundo com o objetivo de colocar os serviços e princípios FAIR em prática.

\section{A iniciativa GO FAIR}

GO FAIR é uma dessas iniciativas internacionais que adota uma abordagem "bottom-up”, ou seja, com atuação dos seus membros de baixo para cima, iniciada com o apoio dos governos da Holanda, Alemanha e França, com a participação dos Estados Unidos, África e Brasil. Seu principal objetivo é ajudar na disseminação e implantação dos produtos e serviços FAIR em todo o mundo.

Suas atividades planejadas estão estruturadas em três pilares:

- GO-CHANGE, que visa tornar os princípios FAIR um padrão de trabalho na ciência, além de reformar os sistemas de recompensa de modo que a avaliação das carreiras de pesquisa reconheça as atividades de ciência aberta; 
- GO-TRAIN, que promove treinamento dentro e fora da Europa, de modo a localizar, criar, manter e sustentar o conhecimento sobre o gerenciamento de dados;

- GO-BUILD, que visa criar infraestruturas para dados interoperáveis, criando padrões, protocolos e serviços compatíveis, possibilitando que os pesquisadores depositem, acessem e analisem dados científicos de todas as áreas (Henning, Ribeiro, Santos, et al., 2019).

\section{GO FAIR Brasil}

A formalização da iniciativa GO FAIR Brasil aconteceu durante o evento dos 20 anos da Rede Scientific Eletronic Library Online (SciELO), quando ficou acordado que o instituído o Instituto Brasileiro em Informação Científica e Tecnológica (IBICT) abrigaria o escritório do GO FAIR no Brasil por possuir uma ampla experiência na implementação de infraestruturas de apoio à informação científica e tecnológica e na formação de políticas de Ciência Aberta.

A GO FAIR Brasil conta com as seguintes instituições parceiras: SciELO, Fundação Oswaldo Cruz (Fiocruz/ICICT), Comissão Nacional de Energia Nuclear (CNEN), Universidade Federal do Estado do Espírito Santo (UFEES) e Universidade Federal do Estado do Rio de Janeiro (UNIRIO) e Universidade Federal do Rio de Janeiro (UFRJ). A rede se compromete com as seguintes atividades:

- Apoiar e coordenar as Redes de Implementação, de acordo com seus objetivos específicos, a adotar as estratégias de implementação dos princípios FAIR aprovadas pela iniciativa GO FAIR;

- Apoiar e coordenar Redes de Implementações que estejam dispostas a definir estratégias de implementação dos princípios FAIR nos casos em que não existam;

- Sistematizar as diretrizes existentes e as criadas pelas Redes, garantindo que elas estejam condizentes com os princípios FAIR;

- Desenvolver mecanismos de difusão das diretrizes definidas pelas Redes de Implementação;

- Manter constante comunicação com o Escritório Internacional de Apoio e Coordenação GO FAIR Internacional (GO FAIR, 2019).

\section{GO FAIR Brasil Saúde}

Em 2018, durante o $10^{\circ}$ Congresso Regional de Informação em Ciências da Saúde (CRICS10), foi formada a primeira rede do GO FAIR, a rede de implementação GO FAIR Brasil Saúde. Está é uma rede temática responsável pela elaboração de estratégias de implementação dos princípios FAIR, no campo da saúde. Sua coordenação está sob a responsabilidade do Instituto de Comunicação e Informação Científica e Tecnologia em Saúde (ICICT), da Fundação Oswaldo Cruz (Fiocruz), que conta com a participação de diversas instituições das áreas de Saúde Pública, Vigilância Sanitária, Informação e Comunicação em Saúde, História do Patrimônio Cultural das Ciências e da Saúde, Oncologia, Enfermagem e Educação Profissional em Saúde (Veiga \& Queiroz, 2019).

Os objetivos da rede são os seguintes:

1) realizar um levantamento dos metadados dos dados de pesquisa, da área da saúde, nacionais e internacionais;

2) desenvolver metodologias para a geração de metadados, alinhados aos princípios FAIR, que atendam às necessidades disciplinares e operacionais da área da saúde;

3) realizar um levantamento dos padrões de interoperabilidade existentes, nacionais e internacionais, da área da saúde, com a finalidade de promover a interoperabilidade tecnológica e semântica dos dados, de acordo com as especificidades dos diferentes domínios da área da saúde;

4) elaborar um Plano de Gestão de Dados geral, totalmente FAIR, para atender as necessidades de gestão dos dados de pesquisa na área da saúde, que sirva de modelo para a Rede GO FAIR Brasil Saúde;

5) realizar um estudo dos principais repositórios de dados de pesquisa, da área da saúde, nacionais e internacionais, e verificar o seu alinhamento com os princípios FAIR;

6) promover o uso, a elaboração e a tradução de padrões voltados para a área da saúde;

7) promover reuniões, cursos, workshops e seminários visando alavancar e disseminar os princípios FAIR entre os seus membros (Veiga \& Queiroz, 2019).

\section{GO FAIR Brasil Saúde Enfermagem}

A Rede GO FAIR Brasil Saúde - Enfermagem, uma sub-rede da GO FAIR Brasil Saúde, é coordenada pelo Programa de Pós-Graduação em Saúde e Tecnologia no Espaço Hospitalar (PPGSTEH), em gestão colegiada 
com o Programa de Pós-Graduação em Enfermagem e Biociências (PPGENFBIO) e o Programa de PósGraduação em Enfermagem (PPGENF), da Universidade Federal do Estado do Rio de Janeiro (UNIRIO).

Seu lançamento ocorreu em setembro de 2020 em seminário online, com a participação das Coordenadoras-Gerais da GO FAIR Brasil e da Rede GO FAIR Brasil Saúde, durante as comemorações do I Congresso Internacional da Escola de Enfermagem Alfredo Pinto, a mais antiga escola de enfermagem do Brasil, que completou 130 anos de existência. Esse lançamento deu início às atividades entre elas uma parceria dos Programas de Pós-graduação em Enfermagem da UNIRIO com o Instituto de Comunicação e Informação em Saúde (ICICT) da FIOCRUZ (ICICT/FIOCRUZ, 2020).

A proposta da rede é atuar pelo fortalecimento e disseminação dos princípios FAIR no campo da enfermagem, de forma articulada e colaborativa com os seus membros, seguindo os mesmos objetivos estabelecidos pela Rede GO FAIR Brasil Saúde, só que restritos ao campo da Enfermagem. Além disso, vem se organizando na promoção de capacitação e cultura relacionada a temática.

\section{Considerações finais}

Podemos considerar que o compartilhamento de dados de pesquisa, neste momento atual de pandemia da COVID-19 é fundamental em todas as áreas do conhecimento e que, na área da saúde, essa prática pode salvar vidas. Além disso, colabora para prevenir e/ou reduzir a possibilidade de perda de informação e agiliza o processo na geração de novos conhecimentos. Tanto os pesquisadores como as instituições acadêmicas e de pesquisa devem gerenciar e preservar seus dados de forma adequada para que a sua reutilização se dê de maneira segura. Para isso, existem técnicas como as já mencionadas neste artigo, tais como, respeitar o ciclo de vidas dos dados e adotar os princípios FAIR, consideradas essenciais para garantir que a boa gestão dos dados aconteça de forma apropriada.

Dados de pesquisa bem organizados são mais fáceis de serem compartilhados e reusados, causando maior impacto, visibilidade e credibilidade não só para o autor, como também para a pesquisa e para a sua instituição.

A coordenação dos Programas de Pós-graduação em Enfermagem da UNIRIO e da Escola de Enfermagem Alfredo Pinto, da Rede GO FAIR Brasil Saúde - Enfermagem representa um exemplo de adoção de práticas da ciência aberta dentro de cursos de pós-graduação. Portanto, espera-se que por intermédio dos estudos e eventos que se iniciam no âmbito da Rede GO FAIR Brasil Saúde - Enfermagem, realizados em 2020 e programados para os anos seguintes, responder a pergunta feita na introdução deste artigo e contribuir para a promoção e implementação de estratégias de capacitação de aluno e profissionais da área da saúde, em geral, sobre a gestão de dados de pesquisa na área da enfermagem. Acreditamos que a ciência aberta é o caminho apropriado a ser seguido pelos pesquisadores da área da enfermagem para tornarem suas investigações mais eficientes na geração de novos conhecimentos e mais seguras, nas tomadas de decisões em novas ações a favor da saúde pública no país.

\section{Referências}

Bertin, P. R. B., Visoli, M. C., \& Drucker, D. P. 2017. A gestão de dados de pesquisa no contexto da e-science: benefícios, desafios e oportunidades para organizações de P\&D . Ponto de Acesso, 11(2), 48.

Instituto Brasileiro de Informação em Ciência e Tecnologia [IBICT]. 2020. Plano de dados abertos: Janeiro/2020-Janeiro/2022.

Brasil, Universidade Federal do Estado do Rio de Janeiro. 2018. Resolução $n^{0}$ 5.055, de 10.10.2018 - PróReitoria de Pós-Graduação e Pesquisa. Disponível em: http://www.unirio.br/propg/resolucoes/resolucao-no-5-055-de-10-10.2018/view

Carvalho, A. V., \& Barbosa Neto, P. A. 2020. Desafios e perspectivas em gestão da informação e do conhecimento. Disponível em: https://repositorio.ufrn.br/jspui/bitstream/123456789/29754/1/Desafios e perspectivas em gestão da informação e do conhecimento.pdf

Cavalcanti, M. T., \& Sales, L. F. 2017. Gestão de dados de pesquisa: um panorama da atuação da União Europeia. BIBLOS, 31(1), 73-98.

Costa, S. M. S. 2006. Open philosophy, business models and funding agencies: Essential elements for the discussion of open access to scientific information. Ciência Da Informação, 35(2), 39-50. 
Publications and Open Access to Research Data in Horizon 2020. Disponível em: https://ec.europa.eu/research/participants/data/ref/h2020/grants_manual/hi/oa_pilot/h2020-hi-oapilot-guide_en.pdf

Fiocruz. 2021. Arca: repositório institucional da Fiocruz - SUS - Brasil. Retrieved May 25, 2021, from https://portal.fiocruz.br/repositorio-institucional-arca

GO FAIR. 2019. GO FAIR Brazil Declaration (EN). Disponível em: https://www.go-fair.org/wpcontent/uploads/2019/06/Declaration_GO-FAIR-Brazil_Jun2019.pdf

Henning, P. C., Ribeiro, C. J. S., Sales, L. F., Moreira, L. R., \& Santos, L. O. B. da S. 2019. Desmistificando os princípios FAIR: conceitos, métricas, tecnologias e aplicações inseridas no ecossistema dos dados FAIR. Pesquisa Brasileira Em Ciência Da Informação e Biblioteconomia, 14(3), 175-192.

Henning, P. C., Ribeiro, C. J. S., Santos, C. J. S., \& Santos, P. X. 2019. GO FAIR e os princípios FAIR: o que representam para a expansão dos dados de pesquisa no âmbito da Ciência Aberta. Em Questão, 25(2), 389-412. https://doi.org/10.19132/1808-5245252.389-412

ICICT/Fiocruz. 2020. Seminário virtual marca o lançamento da Rede GO FAIR Brasil Saúde Enfermagem. Disponível em: https://portal.fiocruz.br/noticia/seminario-virtual-marca-o-lancamento-da-rede-gofair-brasil-saude-enfermagem

Instituto Brasileiro de Informação em Ciência e Tecnologia [IBICT]. 2016. Ibict lança Manifesto de Acesso Aberto a Dados da Pesquisa Brasileira para Ciência Cidadã. https://ibict.br/sala-deimprensa/noticias/item/478-ibict-lanca-manifesto-de-acesso-aberto-a-dados-da-pesquisa-brasileirapara-ciencia-cidada.

Instituto Brasileiro de Informação em Ciência e Tecnologia [IBICT] - Repositórios Digitais. 2018. Disponível em: https://ibict.br/informacao-para-a-pesquisa/repositorios-digitais

Monteiro, G., \& Lucas, E. R. D. O. 2019. Dados científicos abertos: identificando o papel das políticas de gestão e das agências de fomento. AtoZ: Novas Práticas Em Informação e Conhecimento, 8(1), 20.

Nielsen, M. 2012. Reinventing Discovery: The New Era of Networked Science. Princeton University Press.

Raszewski, R., Goben, A. H., Bergren, M. D., Jones, K., Ryan, C., Steffen, A. D., \& Vonderheid, S. C. 2021. A survey of current practices in data management education in nursing doctoral programs. Journal of Professional Nursing, 37(1), 155-162.

Torino, E., Roa-Martínez, S. M., Borsetti, S. A., \& Vidotti, G. 2020. Dados de pesquisa: disponibilização ou publicação? In M. Shintaku, L. F. Sales, \& M. (org) Costa (Eds.), Tópicos sobre dados abertos para editores científicos. ABEC.

Veiga, V. S. de O., \& Queiroz, C. F. de. 2019. Rede GO FAIR Brasil Saúde: uma rede de apoio à Gestão e Abertura de Dados de Pesquisa em Saúde no Brasil. Encontro Ibérico EDICIC. Disponível em: https://www.arca.fiocruz.br/handle/icict/34121

Veiga, V. S. de O., Henning, P., Dib, S., Penedo, E., Lima, J. D. C., Silva, L. O. B. da, \& Pires, L. F. 2019. Plano de gestão de dados fair: uma proposta para a Fiocruz | Fair data management plan: a proposal for Fiocruz. Liinc Em Revista, 15(2), 275-286.

Wilkinson, M. D., Verborgh, R., Silva Santos, L. O. B., Clark, T., Swertz, M. A., Kelpin, F. D. L., Gray, A. J. G., ... Dumontier, M. 2017. Interoperability and FAIRness through a novel combination of Web technologies. PeerJ Computer Science, 2017(4), e110.

\section{Minicurrículo}

Tatiana Souza da Silva Werle. Mestranda do Programa de Pós-Graduação em Saúde e Tecnologia no Espaço Hospitalar da Universidade Federal do Estado do Rio de Janeiro -UNIRIO. Pós-Graduação em Auditoria em Saúde pela Universidade Estácio de Sá, Residência em Clínica Médica e Cirúrgica pela Universidade Federal do Estado do Rio de Janeiro. Graduada em Enfermagem pela Universidade Federal do Estado do Rio de Janeiro. Oficial Enfermeira da Marinha do Brasil atuando como Encarregada da Divisão Técnico Operacional do Departamento de Auditoria de Contas Médicas. Premida como Auditora Honorária pelo Centro de Controle Interno da Marinha. Enfermeira Intensivista do Hospital Moacyr Rodrigues do Carmo e Professora de cursos preparatórios para concursos militares. 
Iana Paes D'Assumpção Vital. Mestranda em Saúde e Tecnologia no Espaço Hospitalar pela Universidade Federal do Estado do Rio de Janeiro (UNIRIO). Pós-graduada em Gestão em Saúde pela Universidade do Estado do Rio de Janeiro (Instituto de Medicina Social - IMS/UERJ). Pós-graduada em Fisioterapia em UTI Adulto pela Interfisio. Graduada em Fisioterapia pela IBMR Laureate International Universities. Graduada no curso técnico profissionalizante em Massoterapia pela IBMR Laureate International Universities. Especialista no Método Acaio de massagem terapêutica para coluna vertebral. Atualmente, Fisioterapeuta Plantonista da UTI Pós-Operatória do Hospital Copa D'Or e Fisioterapeuta Rotina do CTI Adulto do Instituto Estadual do Cérebro Paulo Niemeyer.

Rodrigo Viegas Moure. Mestrando em Saúde e Tecnologia no Ambiente Hospitalar pela UNIRIO. Possui graduação em Arquitetura e Urbanismo pelo Centro Universitário Anhanguera de Niterói (2015) e graduação em Educação Física pela Universidade Estácio de Sá (2009). Atualmente é arquiteto - Octa Arquietura, professor de ginástica coletiva da Academia Tio Sam Barreto e treinador pro - Les Mills Brasil. Tem experiência na área de Educação Física, com ênfase em Educação Física.

Vânia Andréa Schenkel Meireles. Mestrando em Saúde e Tecnologia no Ambiente Hospitalar pela UNIRIO. Atualmente é enfermeira do Instituto Nacional de Câncer.

Debora Daniela Eira Guidi. Mestrando em Saúde e Tecnologia no Ambiente Hospitalar pela UNIRIO. Possui graduação em ENFERMAGEM E OBSTETRICIA pela Universidade Federal Fluminense (1997). Atualmente é enfermeira do Instituto Nacional de Câncer. Tem experiência na área de Enfermagem, com ênfase em Enfermagem em Centro Cirúrgico e Oncologia.

Danielle Guaudino de Paula. Possui graduação em Enfermagem pela Universidade Federal do Estado do Rio de Janeiro (2004), Residência em Enfermagem em Clínica e Cirúrgica (Marinha do Brasil e UNIRIO) (2007), Mestrado em Enfermagem pela Universidade Federal do Rio de Janeiro (2009) e Doutorado em Enfermagem pela Universidade Federal do Rio de Janeiro (2015). Atualmente é professora adjunta da Universidade Federal do Estado do Rio de Janeiro. Coordenadora do Programa de Pós-Graduação Saúde e Tecnologia no Espaço Hospitalar - Mestrado Profissional (PPGSTEH/UNIRIO); Membro Representativo do PPGSTEH junto a Câmara do Núcleo de Inovação e tecnologia da UNIRIO. Tem experiência na área Enfermagem na Saúde do Adulto e do Idoso e Tecnologias no campo da saúde.

Patricia Henning. Pós-Doutora pela University of Twente, na Holanda em 2018/2019. Doutora em Informação e Comunicação em Saúde pelo Instituto de Comunicação e Informação Científica e Tecnológica em Saúde (ICICT/FIOCRUZ) em 2013. Mestre em Ciência da Informação pela Universidade Federal do Rio de Janeiro (UFRJ/IBICT) em 1993. Graduada em Relações Internacionais pela Universidade de Brasília (UNB) em 1986. Professora permanente do Programa de Pós-Graduação em Biblioteconomia (PPGB) da Universidade Federal do Estado do Rio de Janeiro (UNIRIO). A pesquisa de Pós-Doutorado foi sobre a avaliação do nível de FAIRness de Planos de Gestão de Dados Europeus, apoiada pela iniciativa GO FAIR Internacional. É representante do GO FAIR Brasil na Holanda. Professora visitante do Programa de Pós-Graduação em Saúde e Tecnologia no Espaço Hospitalar.

Viviane Veiga. Doutora em Ciências - área de concentração: Informação e Comunicação em Saúde pelo Programa de Pós-Graduação em Informação e Comunicação em Saúde/Fiocruz , com período sanduíche em Universidade de Coimbra (2017). Mestre em Ciências - área de concentração: Gestão da Informação e Comunicação em Saúde pela Escola Nacional de Saúde Pública Sérgio Arouca-Fiocruz (2005). Bacharel em Biblioteconomia e Documentação pela Universidade Federal do Estado do Rio de Janeiro (1999). Atua como pesquisadora na Fundação Oswaldo Cruz, como Editora Associada da Revista Eletrônica de Comunicação, Informação \& Inovação em Saúde (RECIIS). Atua, ainda, como Professora convidada do Programa de PósGraduação em Informação e Comunicação em Saúde (PPGICS/ICICT/Fiocruz) desde 2018, onde ministra 
disciplina obrigatória e eletiva. Atua como professora convidada no Programa de Pós-Graduação em Saúde da Criança e da Mulher, desde 2016. Atua como professora e coordenadora de módulo no Curso de Especialização em Informação Científica e Tecnológica em Saúde (ICTS/ICICT/Fiocruz) desde 2014. Coordena disciplina eletiva no curso de Especialização em Saúde do Trabalhador e Ecologia Humana (ENSP/Fiocruz). Coordena a Rede de Bibliotecas Fiocruz. Coordena a Rede GO FAIR Brasil Saúde. Coordena e participa de grupos de pesquisa em Informação Científica e Tecnológica. Ministra aulas e palestras sobre Fundamentos Teóricos da Informação Científica; Comunicação Científica; Avaliação da Ciência; Ciência Aberta; Acesso Aberto; Dados de Pesquisa; Plano de Gestão de Dados; Princípios FAIR; Repositórios digitais; Data Paper, sistemas de informação; e compartilhamento de dados de pesquisa.

Como citar: Werle, T.S.S., Vital, I.P.D’A., Moure, R.V., Meireles, V.A.S., Guidi, D.D.E., Paula, D.G., Henning, P., \& Veiga, V. 2021. Gestão de Dados de Pesquisa: um desafio para os Programas de Pós-Graduação em Enfermagem. Pubsaúde, 6, a156. DOI: https://dx.doi.org/10.31533/pubsaude6.a156

Recebido: 13 abr. 2021.

Revisado e aceito: 22 abr. 2021.

Conflito de interesse: os autores declaram, em relação aos produtos e companhias descritos nesse artigo, não ter interesses associativos, comerciais, de propriedade ou financeiros que representem conflito de interesse.

Licenciamento: Este artigo é publicado na modalidade Acesso Aberto sob a licença Creative Commons Atribuição 4.0 (CC-BY 4.0). 